Anales de Geografía de la Universidad Complutense ISSN: 0211-9803

https://dx.doi.org/10.5209/aguc.79342

\title{
Cartografía de la hospitalidad en los caminos de peregrinación: el proyecto Mapping Hospitals
}

\author{
Estefanía López Salas ${ }^{1}$ \\ Recibido: 11 de octubre del 2020 / Enviado a evaluar: 15 de diciembre del 2020 / Aceptado: 10 de diciembre del 2021
}

Resumen. A través del uso combinado de herramientas digitales y fuentes documentales históricas, el proyecto Mapping Hospitals plantea un estudio de los hospitales existentes en territorio gallego a mediados del siglo XVIII, al servicio de los peregrinos hacia Santiago de Compostela, con una doble finalidad. Por un lado, proponemos una aproximación de conjunto, es decir, nuestro foco de atención no está en arquitecturas individuales que destaquen por sus características histórico-artísticas, sino en la totalidad de la red de edificaciones con fines caritativos, en una época en la que, además, las peregrinaciones no fueron tan importantes como en la Edad Media, pero sí existieron. Por otro, el proyecto tiene por objetivo el crear un mapa digital que dé acceso abierto a sus resultados a todo el público interesado y que, en sí mismo, se convierta en una herramienta en constante desarrollo hacia nuevos descubrimientos en torno a la hospitalidad y la peregrinación, por medio del análisis asistido por ordenador y la presentación web interactiva.

Palabras clave: Arquitectura hospitalaria; Camino a Santiago; Sistema de Información Geográfica; Mapeado Web.

\section{[en] Cartography of Hospitality on Pilgrimage Routes: The Mapping Hospitals Project}

\begin{abstract}
By combining digital methods with textual sources, the Mapping Hospitals project seeks to study hospitals located in Galicia in the mid-eighteenth century that offered accommodation and care to pilgrims to Santiago de Compostela, with a twofold purpose. On the one side, we aim to approach hospitals as a whole. That is, we are not focused on individual architectures that stand out due to its historical or artistic features, but on the network of buildings that provided charitable hospitality in a time period when this pilgrimage was not as important as in the Middle Ages, but it did existed. On the other hand, we propose to create a digital map to offer open access to the project outcomes to all people who may be interested. The digital map tries to become not only a means of dissemination, but also a tool for an ongoing research towards new discoveries around hospitality and pilgrimage in the St. James Way by means of computer-aided analysis and interactive web-based presentation.
\end{abstract}

Keywords: Pilgrim Hospital Architecture; St. James Way; Geographic Information System; Web-based Mapping

1 Departamento de Proyectos Arquitectónicos, Urbanismo y Composición, Universidade da Coruña. (España).

E-mail: estefania.1salas@udc.es 


\section{[fr] Cartographie de l'hospitalité sur les routes de pèlerinage: le projet Mapping Hospitals}

Résumé. À travers l'utilisation combinée d'outils numériques et de sources documentaires historiques, le projet Mapping Hospitals propose une étude des hôpitaux existants sur le territoire galicien au milieu du XVIIIe siècle, au service des pèlerins de Saint-Jacques-de-Compostelle, dans un double objectif. D'une part, nous proposons une approche globale, c'est-à-dire que notre focus n'est pas sur les architectures individuelles qui se démarquent par leurs caractéristiques historico-artistiques, mais sur l'ensemble du réseau de bâtiments à but caritatif, à une époque où De plus, les pèlerinages n'étaient pas aussi importants qu'au Moyen Âge, mais ils existaient. D'autre part, le projet vise à créer une carte numérique qui donne un accès libre à ses résultats à tout public intéressé et qui, en soi, devient un outil en constante évolution vers de nouvelles découvertes autour de l'hospitalité et de l'hospitalité. pèlerinage, grâce à une analyse assistée par ordinateur et une présentation Web interactive.

Mots-clés: Espagne; mouvements naturels; mouvements migratoires; typologie démographique.

Cómo citar. López Salas, E. (2021): Cartografía de la hospitalidad en los caminos de peregrinación: el proyecto Mapping Hospitals. Anales de Geografía de la Universidad Complutense, 41(2), 389-407.

Sumario. 1. Introducción. 2. De la información al conocimiento a través del interrogatorio moderno. 3. Del catastro de Ensenada a un corpus de arquitectura hospitalaria. 4. Hacia el mapa digital: del texto a la cartografía. 5. El mapa digital on-line Mapping Hospitals. 6. Conclusiones y líneas de trabajo futuras. 7. Referencias bibliográficas.

\section{Introducción}

La noche del 20 de noviembre de 1743, el peregrino italiano Nicola Albani llegaba al Cebreiro tras un largo viaje que había comenzado con su partida de Nápoles, el 11 de junio anterior. El Cebreiro era su primera parada en territorio gallego. Aquí Albani encontró un hospital pequeño del que, en su diario de viaje, dice que en realidad era una palloza atendida por una mujer mayor, con sólo cuatro sacos de paja para dormir disponibles y comida ${ }^{2}$. A su llegada, en este hospital ya se alojaba otro peregrino castellano, que volvía de Santiago. El hombre estaba enfermo desde hacía días y esa misma noche falleció, tras recibir el asilo espiritual del peregrino italiano, por la ausencia del párroco del lugar. Después del Cebreiro, Albani continuó su viaje por los parajes montañosos del camino de Triacastela, en los que se perdió y tuvo grandes dificultades para localizar un alojamiento (Albani, 2007, 139-140; Herbers y Plötz, 1998, 309-311). De hecho, su relato hace especial énfasis en la no existencia de hospederías ni pallozas, hosterías o pensiones en las que un forastero pueda pasar la noche o comprar algo para comer. Muy diferente fue, sin embargo, su experiencia

\footnotetext{
${ }^{2}$ El manuscrito del diario de viaje de Nicola Albani fue publicado en castellano por González, 1993; y en gallego en Albani, 2007. Un extracto del manuscrito correspondiente al tramo gallego también puede consultarse en: Diarios de peregrinos en el Camino de Santiago (en línea), disponible en $<$ http://diariosdeperegrinos.dx.am/nicola.albani.htm $>$ [Consulta: 18/8/2020].
} 
cuando llegó a Portomarín, pueblo en el que destaca que encontró el primer hospital del reino de Galicia con un poco de comodidad, donde le facilitaron un lecho de paja en el que dormir además de limosna. La siguiente jornada terminó en Melide buscando alojamiento en una palloza por caridad. San Marcos fue su última parada antes de llegar a Santiago de Compostela. De nuevo, en este caso su relato se focaliza en plasmar la experiencia de la dificultad de encontrar a alguien que le diera cobijo. Al estar lloviendo, relata que se vio obligado a hacer de predicador, casa por casa, hasta que un vecino salió y lo llevó a su palloza, en la que pasó la noche a gusto (Albani, 2007, 139-140).

Fue el día 25 de noviembre de 1743 cuando Nicola Albani llegó a su destino, Santiago de Compostela, en donde permaneció hasta el 11 de diciembre. Primeramente, se alojó en el Hospital Real de la ciudad, en el que le asignaron un lecho poco limpio que no incluía la cena, aunque el comer bien reconoce que sí estaba garantizado en los refectorios de los distintos conventos urbanos (Albani, 2007, 149150; Herbers y Plötz, 1998, 317). Asimismo, indica que en este edificio sólo se daba alojamiento durante tres noches, la primera de las cuales compartió techo con unos ciento sesenta peregrinos de diferentes países, dejando testimonio también de que todos los días llegaban treinta o cuarenta más (Albani, 2007, 142). Pasadas esas tres primeras noches de hospedaje gratuito, buscó habitación en una posada regentada por una mujer en la que había otros dos peregrinos. Según Albani en la ciudad vivían numerosas mujeres que se dedicaban a alquilar habitaciones o camas a peregrinos, todas con colchones de paja, por un módico precio, pero que también existían otras más cómodas y limpias para personas de mayor rango social.

El anterior es un pequeño extracto de las vivencias del italiano Nicola Albani en su peregrinación a Compostela que, nos es conocida, gracias al diario que él mismo escribió sobre su periplo, Veridica Historia o' sia Viaggio da Napoli à S. Giacomo di Galizia (González, 1993; Albani, 2007). Este es uno de los mejores testimonios literarios de la pervivencia de la peregrinación jacobea en el siglo XVIII (González, 2018, 46; Arribas, 2001, 6; Herbers y Plötz, 1998, 299-322), pero también un emotivo reflejo de cómo los hospitales, su presencia y ausencia en los caminos, seguían siendo determinantes en la experiencia del peregrino bien avanzada la Edad Moderna (Lois y López, 2015, 146-147, 151-152). El relato de Albani no sólo nos habla de ellos como edificios de los que dependía el cobijo, sino también la comida, la bebida, la limosna, las atenciones médicas en el caso de caer enfermo, la asistencia espiritual e incluso el entierro si la muerte los sorprendía de camino. En otras palabras, en este texto reconocemos la persistencia del concepto medieval de hospital, que estaba próximo a cambiar en la segunda mitad del siglo XVIII, así que los estados se hicieron cargo de una reforma hospitalaria, de acuerdo con las ideologías ilustradas y unas nuevas condiciones sociales y económicas (García, 1996, 21; Gijón, 2017-2018, 263-264).

El proyecto Mapping Hospitals nace con la intención de estudiar el conjunto de hospitales al servicio de los peregrinos hacia Santiago de Compostela, dispersos por todo el territorio gallego, a mediados del siglo XVIII, a través de su visualización en un mapa digital que no sólo haga accesible los resultados de la investigación a un público amplio interesado, sino que también abra el camino hacia nuevos 
descubrimientos en torno a la hospitalidad y la peregrinación jacobea ${ }^{3}$. Si bien mantenemos el estudio del hospital en su concepto medieval, abordando el conjunto de edificios frecuentados por pobres, marginados, peregrinos, enfermos y viajeros, el periodo temporal en el que este estudio se enfoca no es el de mayor concentración de las peregrinaciones, sino el de mediados del siglo XVIII que, tradicionalmente, ha quedado fuera del principal foco de atención de los estudiosos, por la disminución que aquellas experimentaron en la Edad Moderna. Asimismo, frente una visión de realidades individuales, se plantea un cambio de escala, una aproximación global, conectada, comparativa y en relación con el territorio, es decir, el estudio de la red de hospitales al servicio de la caridad, como cambio de lente que nos permita descubrir nuevas realidades, que pueden pasar desapercibidas cuando examinamos las mismas arquitecturas, pero de forma aislada.

\section{De la información al conocimiento a través del interrogatorio moderno}

A lo largo del siglo XVIII, se observa un deseo constante por parte del Estado de conocer la realidad social, económica y cultural del conjunto del territorio castellano, a través de una recopilación sistemática de información (Rodríguez, 2004, 950-958). Entre los instrumentos utilizados con este fin figuraron descripciones de viajes, cuestionarios, encuestas, censos, diccionarios geográficos e interrogatorios, entre otros. El objetivo era averiguar para conocer y, en base a lo recabado, poder llevar a cabo reformas de carácter global. Hoy todos los materiales generados en ese periodo son muy valiosos para aproximarnos a la realidad histórica del espacio sometido a conocimiento.

Dentro de estos procesos informativos modernos de utilidad para el Estado, se engloba la iniciativa de Felipe $\mathrm{V}$ de elaborar un censo de hospitales que, en un paso posterior, permitiese acometer una reforma de conjunto, no sólo para garantizar una mejor atención de los usuarios de aquellos, sino, y sobre todo, para reestructurar su administración en beneficio de la causa pública (Rodríguez, 2004, 962; Gijón, 20172018, 263). Con este objetivo en mente, en la Real Provisión del 22 de enero de 1739, el monarca dio un primer impulso al proceso ordenando una averiguación de datos muy concretos sobre los hospitales existentes en su territorio, en base a un interrogatorio formado por siete preguntas, que debían hacerse a nivel municipal (Rodríguez, 2004, 961-962).

La primera de ellas se preocupaba por recopilar información sobre qué hospitales había en las ciudades y villas de la jurisdicción que realizaba el informe. En segundo lugar, para conocer la realidad material de cada institución hospitalaria relacionada en

\footnotetext{
${ }^{3}$ Este proyecto fue parcialmente financiado por la Cátedra del Camino de Santiago y de las Peregrinaciones de la Universidad de Santiago de Compostela, gracias a la I Beca de Investigación sobre el Camino de Santiago y las Peregrinaciones, concedida en Junio de 2018.
} 
el punto anterior, se interrogaba a los municipios sobre el tipo de fábrica o casa que acogía esta función y cuál era su estado en aquel momento. En el caso de que los edificios estuviesen mal conservados, necesitados de reformas o con las fábricas ruinosas, la pregunta tercera daba cuenta de ello. Asimismo, el interrogatorio pretendía recabar datos sobre los trabajos de reparación necesarios, de acuerdo a la capacidad de la población en la que se localizaban aquellos. En cuarto lugar, cada ámbito territorial tenía que facilitar información relativa al equipamiento de los hospitales, en concreto, a su menaje y mobiliario. Las dos preguntas siguientes se focalizaban en averiguar qué personas ocupaban los edificios (personal, usuarios y asistentes) y, en último lugar, la séptima pregunta ahondaba en qué renta tenía el hospital y cómo era su estado, qué personas administraban el edificio y a quién pertenecía o si este era de patronato.

Estos eran todos aspectos fundamentales para tener un buen conocimiento de la realidad de la red hospitalaria existente en la Corona de Castilla de la primera mitad del siglo XVIII, pero no tenemos constancia de que el censo se llegase a realizar (García, 1996, 21). No obstante, sí contamos con otra fuente documental, el catastro del marqués de la Ensenada que, con la misma intención averiguadora propia de la época, pero con una finalidad muy distinta, es el punto de partida del trabajo hasta ahora desarrollado dentro del proyecto Mapping Hospitals.

El 10 de octubre de 1749, siendo ministro de Hacienda don Zenón de Somodevilla y Bengoechea, I marqués de la Ensenada, el rey Fernando VI aprobaba el decreto que ponía en marcha la realización de una reforma fiscal en los territorios de la Corona de Castilla (Camarero, 2002, 63-64). Su finalidad primordial era establecer un solo impuesto que, teniendo en cuenta la capacidad contributiva de cada sujeto, garantizase una carga impositiva equitativa, clave para el saneamiento de la Hacienda pública (Alimento, 2002, 21-22). Para ello, primero era necesario recabar información sobre todos los bienes que cada habitante poseía en el conjunto del territorio afectado. Esto fue posible a través de un trabajo exhaustivo de averiguación, que dio lugar al llamado catastro del marqués de la Ensenada, en honor a su principal impulsor.

En el proceso de realización de este censo global de la riqueza urbana y rústica de la Corona de Castilla de mediados del XVIII, el primer nivel de aproximación a la realidad fue el de la feligresía. Consistió en recabar, de forma sistemática, las respuestas a cuarenta preguntas, establecidas en un Interrogatorio, para cada unidad territorial de catastración. Estas constituyen las Respuestas Generales que, por su volumen y utilidad para el análisis histórico, fueron digitalizadas durante los años 2004 y 2005 y hoy son accesibles a cualquiera interesado en el Portal de Archivos Españoles ${ }^{4}$.

En el estudio que nos ocupa, concentramos la atención en una única pregunta del Interrogatorio, la número 30, en la que intentaban averiguar si, en el ámbito territorial

\footnotetext{
${ }^{4}$ Ministerio de Cultura. Catastro de Ensenada - Portal de Archivos Españoles (PARES) [en línea], disponible en $<$ http://pares.mcu.es/Catastro/ $\geq$ [Consulta: 18/8/2020].
} 
catastrado, existía algún hospital y, de haberlo, cuál era su calidad, renta y modo de mantenerse ${ }^{5}$. Dicho está que el catastro de Ensenada fue realizado para un fin muy distinto del de conocer los edificios destinados a fines caritativos que, por el contrario, sí tenía la real provisión de 1739 emitida por Felipe V, pero, por su necesidad de conocimiento de la realidad de todo tipo de bienes susceptibles de tributo, indirectamente recopiló datos que, si bien dispersos en múltiples manuscritos, son una base de partida única para acceder a la imagen de conjunto, para empezar a convertir la historia de las individualidades en la de las arquitecturas conectadas.

\section{Del catastro de Ensenada a un corpus de arquitectura hospitalaria}

Galicia es el ámbito territorial objeto de estudio en la primera fase de desarrollo del proyecto Mapping Hospitals. Cuando se llevó a cabo el catastro de Ensenada, el Reino de Galicia se dividía en siete provincias (A Coruña, Betanzos, Santiago, Lugo, Mondoñedo, Ourense y Tui) (Castro, 2019) ${ }^{6}$. En su conjunto definían el territorio gallego con unos límites administrativos que, por el este, no incluían algunas poblaciones de las antiguas provincias de Léon y Valladolid, que hoy sí forman parte de la comunidad autónoma de Galicia (Figura 1a). Teniendo en cuenta también estas últimas, la averiguación catastral de mediados del setecientos generó un total de 2912 expedientes de Respuestas Generales en el territorio que nos ocupa, de los cuales 1229 se corresponden con entidades hoy pertenecientes a la provincia de Lugo, 664 a la de A Coruña, 605 a la de Ourense y 414 a la de Pontevedra (Figura 1b). Este es el conjunto de documentos que fue necesario consultar para el alcance de los objetivos aquí planteados.

Cada expediente es un manuscrito, en general de fácil lectura, que recopila las respuestas dadas por una población concreta a las cuarenta preguntas de un cuestionario o interrogatorio común. Si bien, el volumen de documentos a consultar es considerable, razón que influyó en limitar el estudio a Galicia, el trabajo se concentra, como ya señalamos, en la lectura de la respuesta a la pregunta número treinta de cada unidad territorial ${ }^{7}$. Hecho esto, primeramente determinamos que, del total de 2912 expedientes citados, solamente 72 contienen una respuesta positiva a aquella: 3 se corresponden a poblaciones ubicadas en la antigua provincia de A Coruña, 19 en la de Santiago, 11 en la de Betanzos, 18 en la de Lugo, 9 en la de

\footnotetext{
${ }^{5}$ Ministerio de Cultura. Respuestas Generales - Grafía Actual. En Catastro de Ensenada - Portal de Archivos Españoles (PARES) [en línea], disponible en $<$ http://pares.mcu.es/Catastro/> [Consulta: 18/8/2020].

6 Material cartográfico disponible en $<$ https://galiciadigital1753.wixsite.com/proyecto $>$ [Consulta: 20/8/2020].

${ }^{7} \mathrm{La}$ aplicación de metodologías de aprendizaje automático para la lectura de textos manuscritos como las desarrolladas en el proyecto Carabela, disponible en $<$ http://carabela.prhlt.upv.es/es $\geq$, reduciría costes y tiempos de trabajo.
} 
Mondoñedo, 6 en la de Ourense y otras 6 en la Tui (Figura 2a) ${ }^{8}$. De cada documento consultado en el que sí se reconocía la existencia de uno o varios hospitales en la población, realizamos una transcripción completa de la respuesta dada.

Figura 1: (a) División provincial de 1753 en verde y en gris los límites administrativos actuales, según Castro, 2019. (b) Número de libros de Respuestas Generales del catastro de Ensenada generados para cada provincia de la división administrativa actual.

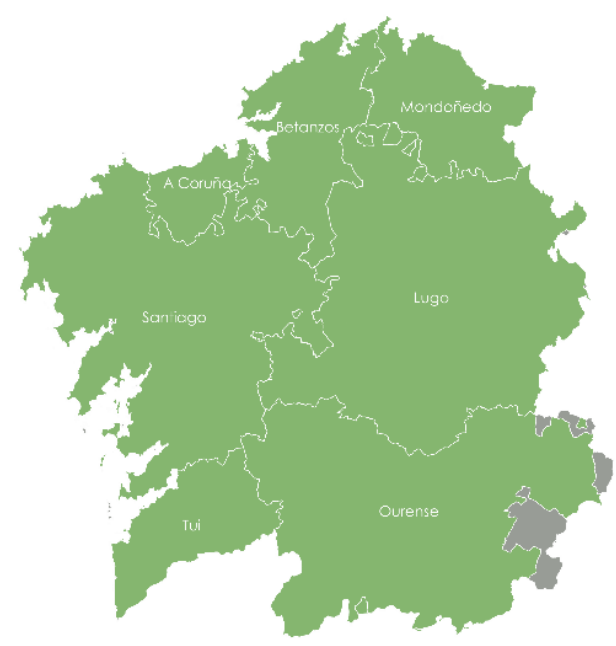

a

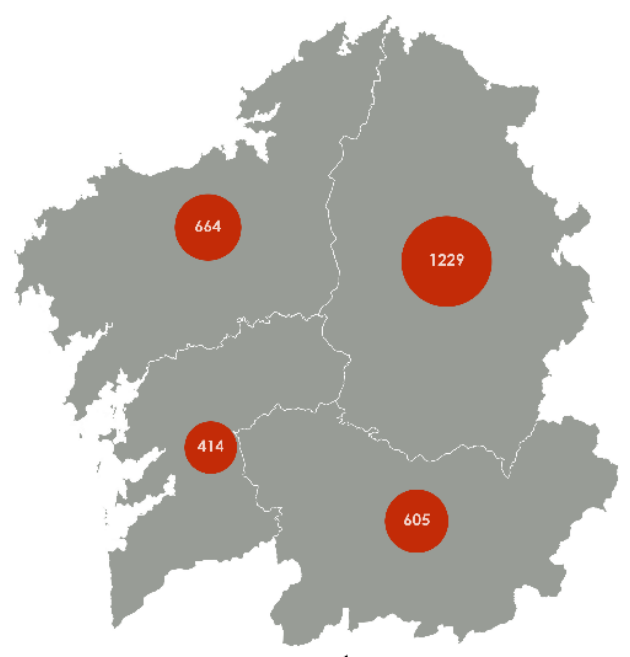

b

Fuente: Elaboración propia.

A partir de esta información, se afrontó un segundo paso del proceso que consistió en la creación de un corpus ordenado de los datos extraídos. Este está formado por un total de 88 arquitecturas hospitalarias localizadas a partir de la lectura de las 72 respuestas positivas del paso previo, teniendo en cuenta que, en cada población catastrada, podían existir una o varias edificaciones con este fin, ya se refiriesen a ellas con el nombre de hospital, hospicio, posada, casa o de otro modo. En cualquier caso, todas responden al concepto de edificación al servicio de la caridad, destinada a acoger a peregrinos de camino a Santiago u otros lugares, pero también pobres locales y extranjeros-, viajeros, enfermos de todo tipo y cualquier otro usuario que no era capaz de cuidar de sí mismo, ya fuera a nivel económico o físico, dentro de la sociedad de la época. De estas 88 arquitecturas, 5 se encontraban en la provincia de A

\footnotetext{
${ }^{8}$ Con respecto a los límites provinciales actuales, se contabilizan 27 respuestas positivas en el caso de la provincia de Lugo, 26 en el de A Coruña, 6 en el de Ourense y 13 en el de Pontevedra.
} 
Coruña, 26 en la de Santiago, 13 en la de Betanzos, 19 en la de Lugo, 11 en la de Mondoñedo, 7 en la de Ourense y otros tantos en la de Tui (Figura 2b) ${ }^{9}$.

Figura 2: (a) Número de libros de Respuestas Generales con informe positivo a la pregunta número 30 del Interrogatorio del catastro de Ensenada. (b) Total de arquitecturas hospitalarias en cada una de las siete provincias del Reino de Galicia.

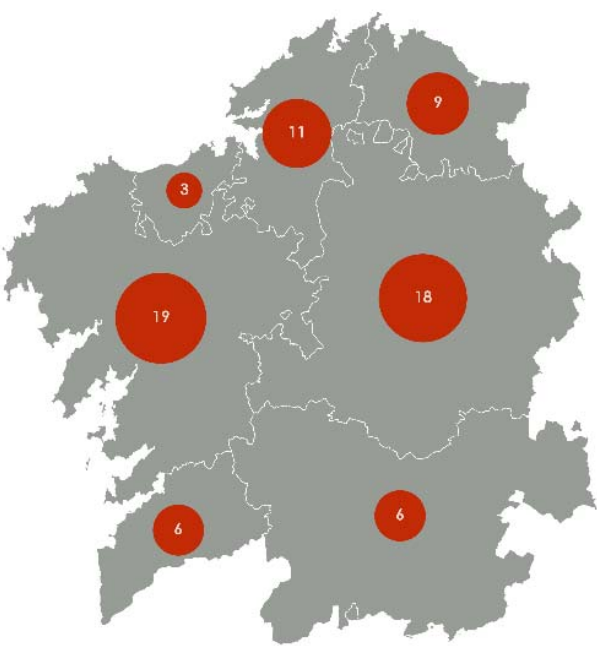

a

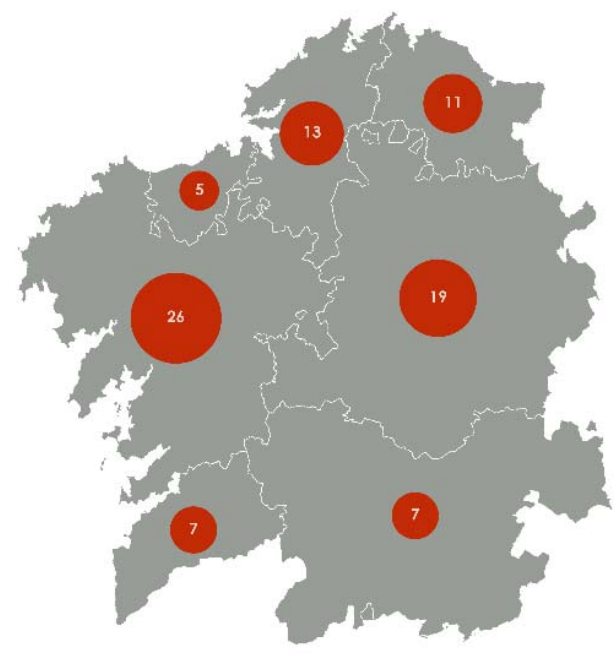

b

Fuente: Elaboración propia.

Cada edificio del corpus es una entidad distinta a la que vinculamos un conjunto de datos, organizados según una estructura clara y común, formada por los ítems siguientes: nombre del hospital, provincia antigua, provincia actual, nombre de la población, usuario, número de camas, fundación, patronato, administrador y capilla asociada. Hay que señalar que estos no se corresponden literalmente con los relacionados en la pregunta 30 del Interrogatorio: existencia de hospital, calidad, renta y modo de mantenerse, porque el carácter ordenado del punto de partida del proceso catastrador no se tradujo en los resultados, en la mayoría de los casos. Como consecuencia, para una misma pregunta, en los manuscritos encontramos una enorme variabilidad de respuestas que no alcanzan, igualan o bien superan el tipo y número de datos que las instrucciones del catastro planteaban. De hecho, los ítems antes relacionados son un reflejo de aquella información concreta que, con mayor frecuencia, aparece en las respuestas dadas y, al mismo tiempo, una selección de la

\footnotetext{
${ }^{9}$ De nuevo, con respecto a los límites provinciales actuales, se contabilizan: 37 arquitecturas hospitalarias en la provincia de A Coruña, 30 en la de Lugo, 7 en la de Ourense y 14 en la de Pontevedra.
} 
que mejor nos puede ayudar a comprender, no tanto cuestiones de carácter fiscal, sino de la arquitectura en sí desde un punto de vista histórico.

Fuera de esta estructura principal del corpus quedan, sin embargo, otros muchos datos importantes para nuestro estudio, pero que con muy escasa frecuencia fueron anotados, como la indicación del lugar de implantación del hospital, dentro de la población, el estado de conservación del edificio, la descripción de su organización interior, etc. Para no perder ninguno de ellos y dejar la puerta abierta a futuras ampliaciones del corpus de partida, el último ítem contiene la transcripción íntegra de la pregunta en la que cada hospital fue informado. Finalmente, aunque este es el primer dato del corpus, a cada arquitectura hospitalaria le asignamos un código, que la identifica de forma inequívoca (Tabla 1).

Tabla 1: Extracto del corpus de arquitecturas hospitalarias de mediados del setecientos elaborado dentro del proyecto Mapping Hospitals.

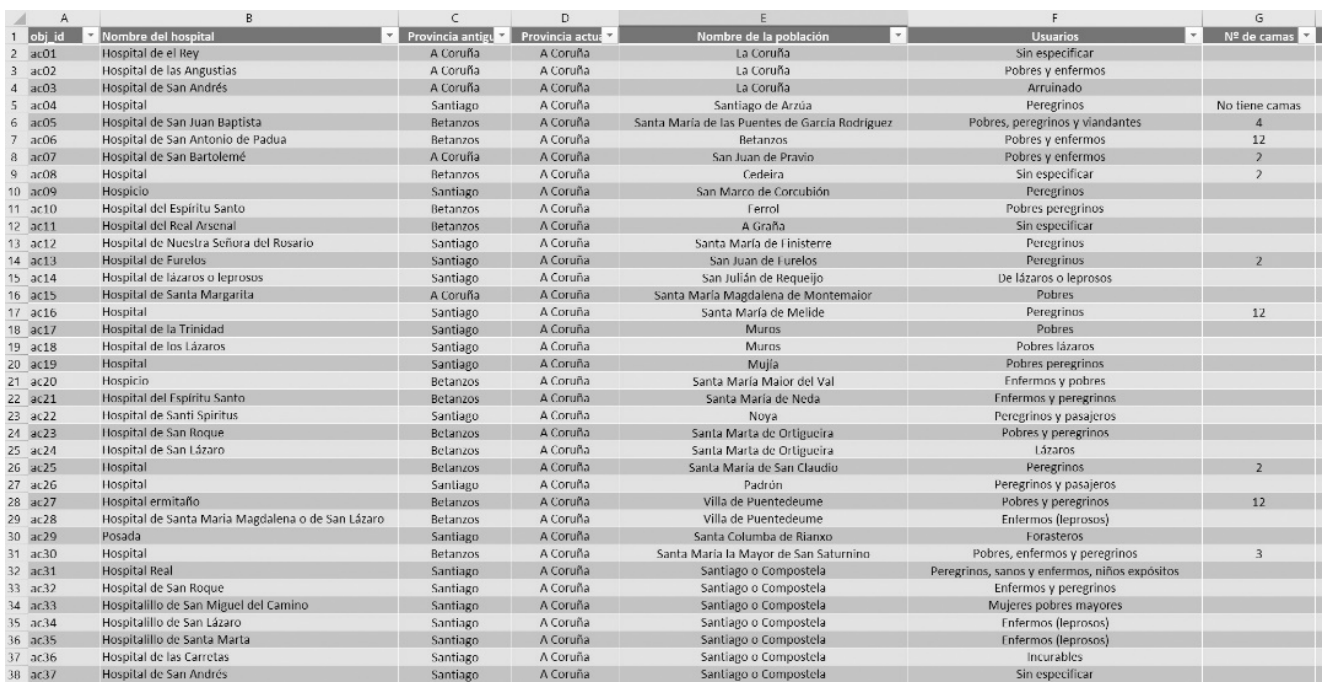

Fuente: Elaboración propia.

La organización de los datos nos permitió detectar tan sólo una construcción hospitalaria repetida entre dos expedientes distintos de la provincia de Lugo, así como obtener unos primeros resultados de la interpretación inmediata de los mismos. En ellos vemos que las antiguas provincias de Lugo y Santiago eran las que disponían de más hospitales, posiblemente porque, no sólo tenían una mayor superficie y número de poblaciones dentro de sus límites, sino también porque eran las que cruzaba de este a oeste el principal camino de peregrinación medieval, el camino Francés, para cuyo servicio nacieron y probablemente pervivieron en el tiempo muchas de estas construcciones. Si continuáramos con el análisis del corpus podríamos responder ya a otras muchas cuestiones de conjunto como: ¿qué poblaciones de la Galicia de mediados del siglo XVIII tenían más de un hospital? ¿cuántos de ellos estaban 
destinados exclusivamente a peregrinos hacia Santiago de Compostela? ¿qué número sólo atendían a enfermos? ¿cuántos eran construcciones especializadas en la atención de un único tipo de usuario, como los leprosos?, etc.

Sin embargo, la creación y análisis de este censo de hospitales del setecientos, no es el objetivo final del trabajo. Lo que aquí nos planteamos es estudiar la red de estos edificios desde una escala geográfica que se aproxime a la hospitalidad a través de una cadena de edificios dispersos en el territorio, como mecanismo para descubrir nuevas cuestiones sobre la arquitectura de la peregrinación, que quedan al margen en un estudio de las individualidades, o desde una aproximación de conjunto que ignora el territorio por no poder leerlo al mismo tiempo.

\section{Hacia el mapa digital: del texto a la cartografía}

El nombre de la población en la que se situaba cada hospital figura entre los datos recolectados en el corpus. En el catastro de Ensenada esta era generalmente una feligresía o parroquia, una villa o una ciudad, lo cual implica un ámbito territorial con diversas localizaciones posibles dentro de sus límites. No obstante, rara vez los peritos llegaron a anotar en las respuestas generales en qué sitio concreto de aquella se ubicaba el hospital u hospitales, porque no era necesario saberlo para los fines fiscales del catastro y, en menos casos aún, acompañaron sus averiguaciones de algún tipo de documento gráfico, como un plano, que hoy pudiera acercarnos a la realidad física del pasado del territorio averiguado.

Por tanto, visualizar las arquitecturas individuales en relación a su posición en el territorio, para obtener la imagen de la red y abrir las puertas hacia el análisis de su conexión con aquel, es un ejercicio que se puede realizar con la información extraída del catastro y el corpus creado a partir de él, pero para una escala de aproximación muy concreta, aquella para la que fue hecha la averiguación. Si, además, combinamos estos datos, que son exclusivamente textuales, con otras fuentes y trabajos previos que nos aporten información sobre la ubicación exacta de cada hospital dentro de la población, la posibilidad de análisis de la relación histórica del edificio con el territorio que le daba soporte puede ser mayor.

Algunos hospitales existentes a mediados del setecientos todavía se conservan en pie en la actualidad. Aunque la mayor parte han sido transformados y su función original modificada, el lugar de ubicación es el mismo que en el siglo XVIII. En otros muchos casos, sin embargo, el hospital no es un elemento permanente en la trama contemporánea. Para este segundo grupo, en ocasiones sabemos en qué lugar concreto del asentamiento o feligresía se situaba el edificio desaparecido, gracias a que contamos con una representación de él en fuentes cartográficas o fotográficas históricas. A partir de la información contenida en estas es posible deducir sus coordenadas en una ortofotografía actual. No obstante, también es cierto que de un número considerable de arquitecturas hospitalarias catastradas en el setecientos, hoy no se conservan huellas materiales y, como mucho, se reducen a una toponimia que hace una mínima referencia a su anterior existencia. Esta nos ayuda a localizar el área 
dentro del asentamiento en la que sus fábricas pudieron levantarse, pero sólo de forma aproximada $^{10}$.

Conocidas las coordenadas de cada arquitectura, procedimos a su georreferenciación haciendo uso de un sistema de información geográfica, QGIS, que nos permite, en primer lugar, visualizarlas sobre el territorio. Esta parte del proceso de trabajo es la antesala del mapa interactivo final. En QGIS, cada hospital se representó como un punto que tiene una serie de atributos asociados. Estos son equivalentes a los mismos ítems del corpus creado a partir del catastro. Cada punto está geolocalizado, es decir, tiene las coordenadas del lugar de ubicación del hospital, que son las exactas si se conocen, o de lo contrario aproximadas de acuerdo a las fuentes disponibles, pero siempre situadas dentro de la población correspondiente y en un área de esta en la que la toponimia, la presencia de alguna capilla o iglesia con un advocación determinada u otros factores, guíen la localización.

El resultado de convertir los datos textuales en elementos gráficos es la obtención de un primer mapa en el que aparecen representadas todas las arquitecturas hospitalarias a través de puntos sobre una base cartográfica actual (Figura 3a). Asimismo, se añadió una capa con la representación de los límites provinciales del Reino de Galicia en 1753, de forma que aquellos se pueden visualizar también en relación con la división administrativa histórica (Figura 3b). Si analizamos estos dos mapas, destaca la concentración de arquitecturas hospitalarias que se producía en las zonas de costa en el siglo XVIII. Hacia el interior del territorio gallego, no sólo había un menor número de estos edificios, sino que también estaban mucho más dispersos. No obstante, los hospitales del interior, en algunos casos, dibujan sobre el territorio rutas claras, caminos, lo que empieza a revelar la persistencia de una red histórica que los conecta. El conjunto de 88 arquitecturas georreferenciadas en QGIS convierte el corpus textual en uno gráfico, base del mapa digital final.

En un primer paso, todas las arquitecturas hospitalarias fueron representadas dentro de una misma capa. No obstante, uno de los objetivos de partida de este proyecto es el estudio del conjunto de hospitales al servicio de los peregrinos hacia Santiago de Compostela y, como consecuencia, también consideramos necesario hacer una visualización de estas arquitecturas de acuerdo al tipo de usuario para el que el catastro indica que estaban destinadas. Así, en un segundo paso se crearon un total de 17 capas. Cada una de ellas nos permite visualizar, de forma independiente, los edificios utilizados por un grupo de usuarios determinado que dan nombre a la capa, incluidos aquellos de los que este dato no fue especificado. Las 7 primeras capas contienen edificaciones que estaban destinadas a acoger peregrinos, hacia Compostela u otros lugares, de forma exclusiva, o bien compartida con otros usuarios (Figura 4a). Estos últimos podían ser viajeros o pobres, pero también enfermos. Un

\footnotetext{
${ }^{10}$ De las 88 arquitecturas catastradas, en base a estudios previos, cartografía y fotografía histórica, hemos podido determinar las coordenadas de ubicación de un total de 60. Por tanto, se hace necesario, en un futuro desarrollo del proyecto, la búsqueda de información para los 28 casos restantes que ayude a completar, del modo más preciso posible, esta cuestión.
} 
total de 16 edificaciones tenían como único usuario a peregrinos, 8 estaban destinadas a acoger peregrinos y pobres, 6 compartían la atención a peregrinos y a enfermos, 4 daban hospedaje a peregrinos y viajeros, otras 4 a peregrinos, pobres y viajeros, e igual número se destinaban a peregrinos, pobres y enfermos. Una menor cantidad de arquitecturas, 2, tenían entre sus usuarios a peregrinos, pobres, enfermos y viajeros, y tan sólo en una edificación se señala que además de los peregrinos se ocupaban de los huérfanos.

Figura 3: (a) Arquitecturas hospitalarias del catastro del setecientos geolocalizadas en QGIS sobre Google Maps, 2020. (b) Arquitecturas hospitalarias del catastro del setecientos

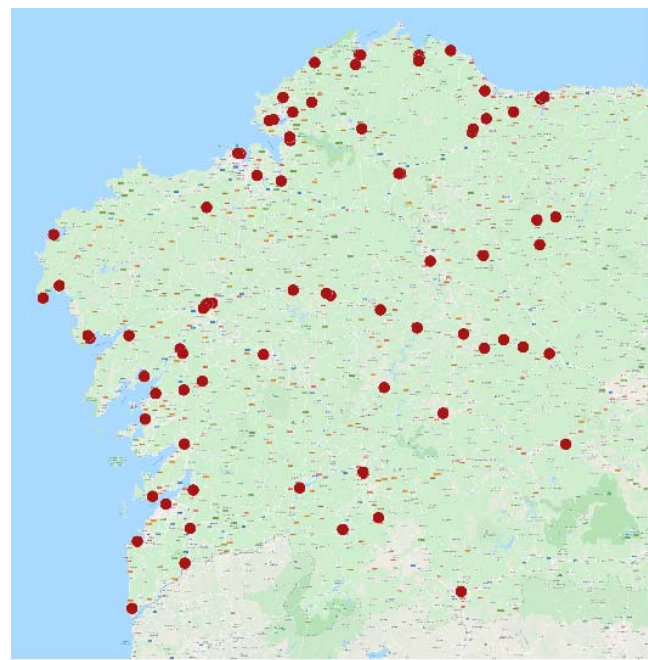

a

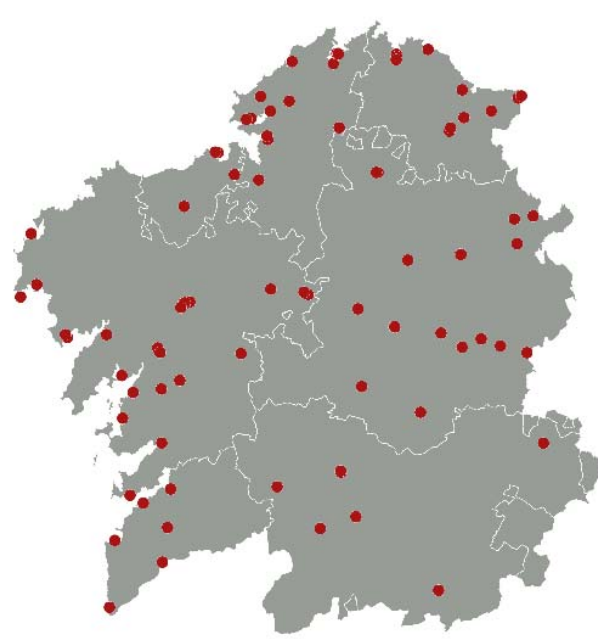

b

Fuente: Elaboración propia.

A continuación, se incluyeron otras 7 capas de hospitales clasificados según diferentes usuarios, pero que comparten el hecho de que entre estos el catastro no cita que figuren los peregrinos ${ }^{11}$. Aquí se encontraba un grupo de 4 fábricas destinadas a atender sólo a enfermos, así como 12 que únicamente atendían a un tipo específico de estos, los lazarosos o leprosos, y 1 a soldados aquejados de alguna dolencia. No obstante, también se contabilizan hasta 6 edificios en los que los enfermos compartían lecho con los pobres. En último lugar, existían arquitecturas que, según los datos

\footnotetext{
${ }^{11} \mathrm{Al}$ menos en un caso de los incluidos en este segundo grupo sabemos que, aunque el catastro no cite que el hospital atendía a peregrinos, sí estaba destinado a ellos. Se trata del hospital de la villa de Samos, en las inmediaciones del monasterio benedictino de San Julián de Samos. Esta misma situación podría darse para otros ejemplos, algo que sólo la utilización de otras fuentes nos permitiría determinar.
} 
averiguados por los peritos, sólo acogían a pobres, en 6 casos, y 1 sólo para forasteros. Gente pobre del lugar o extranjeros compartían estancia en 2 edificaciones. Asimismo, de un total de 11 hospitales el catastro no cita el usuario, bien porque no fue averiguado o bien porque la fábrica se dice que estaba ruinosa y, por tanto, sin uso a mediados del XVIII. Estos se incluyeron en las dos últimas capas creadas en QGIS (Figura $4 b$ ).

Figura 4: (a) Arquitecturas hospitalarias destinadas a acoger peregrinos, de forma exclusiva, o compartida con otro tipo de usuarios a mediados del XVIII. (b) Arquitecturas hospitalarias destinadas a acoger diversos tipos de usuarios entre los que no figuraban peregrinos, incluidos aquellos de los que este dato no se especifica, a mediados del XVIII.

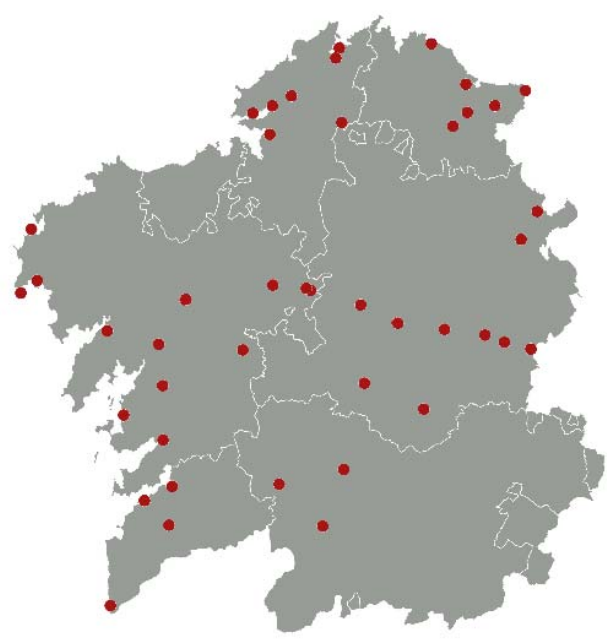

a

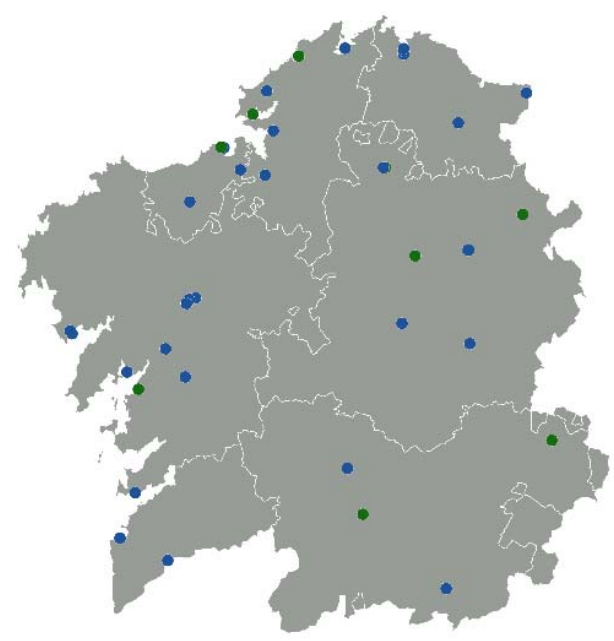

$\mathrm{b}$

Fuente: Elaboración propia.

La visualización y lectura jerarquizada de estos datos, dejando fuera los casos cuyos usuarios el catastro no especificó, nos permite descubrir la persistencia, a mediados del siglo XVIII, de la arquitectura del hospital con un tipo de usuario predominante en el territorio gallego, que es el peregrino, con un total de 44 ejemplos, frente a los 32 destinados a atender a otro tipo de huéspedes (Figura 5). 
Figura 5: Gráfico de rectángulos que visualiza los datos cuantitativos de hospitales jerárquicamente organizados según las tres grandes agrupaciones ${ }^{12}$.

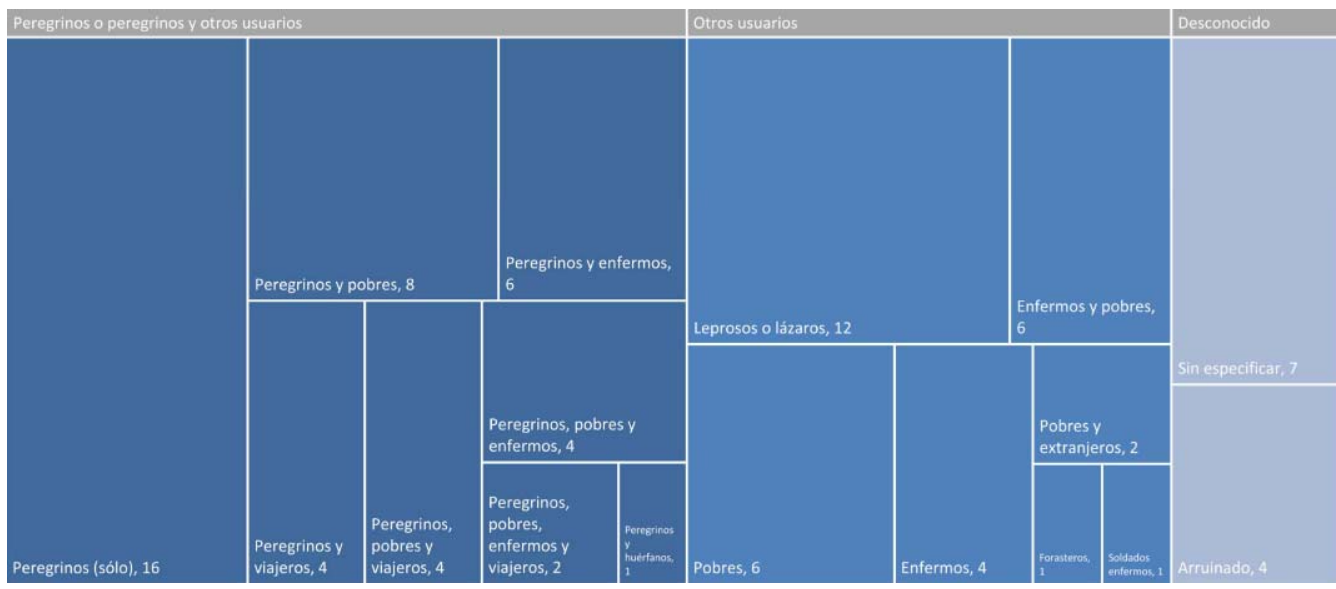

Fuente: Elaboración propia.

No obstante, también es cierto que la atención sanitaria, considerada una acción caritativa desde la Edad Media, estaba presente en un buen número de fábricas, hasta 38 del total.

\section{El mapa digital on-line Mapping Hospitals}

Como señalamos al inicio, la implementación de un mapa digital fue una prioridad desde el comienzo de este proyecto, con una doble finalidad, que este se convirtiera en una herramienta de investigación en sí misma, en torno a la hospitalidad y la peregrinación a Santiago de Compostela, pero también en un medio de difusión de los resultados alcanzados y en constante crecimiento. Para ello, se creó el sitio web $<$ http://mappinghospitals.udc.es/>, cuya primera versión fue lanzada el 27 de mayo del año 2020.

El sitio se organiza en torno a un mapa interactivo con las 88 arquitecturas hospitalarias gallegas representadas como puntos georreferenciados sobre el territorio con un determinado color. El color es un indicativo de pertenencia a alguna de las 18 capas creadas previamente en QGIS, es decir, nos habla de un tipo de usuario o usuarios, de acuerdo a los datos del catastro. Los puntos que representan a cada hospital tienen un tamaño que se escala automáticamente a medida que aumentamos o

\footnotetext{
${ }^{12}$ Gráfico de rectángulos que visualiza los datos cuantitativos de hospitales jerárquicamente organizados según las tres grandes agrupaciones representadas en la cabecera, así como la proporción que, dentro de cada jerarquía, tienen los diferentes usuarios o grupos de usuarios respecto del conjunto.
} 
disminuimos el zoom. Asimismo, su color ha sido asignado con cierto grado de transparencia de modo que, si la escala del plano es muy grande y, como consecuencia, diferentes puntos se superponen entre sí, los demás sigan siendo visibles, al menos parcialmente, y viceversa. A una escala pequeña, la transparencia del color de relleno permite ver en su totalidad el sitio dónde estaba o dónde todavía está la arquitectura en cuestión, a pesar de la superposición.

Figura 6: Inicio del sitio web del proyecto Mapping Hospitals en su versión en castellano ${ }^{13}$.

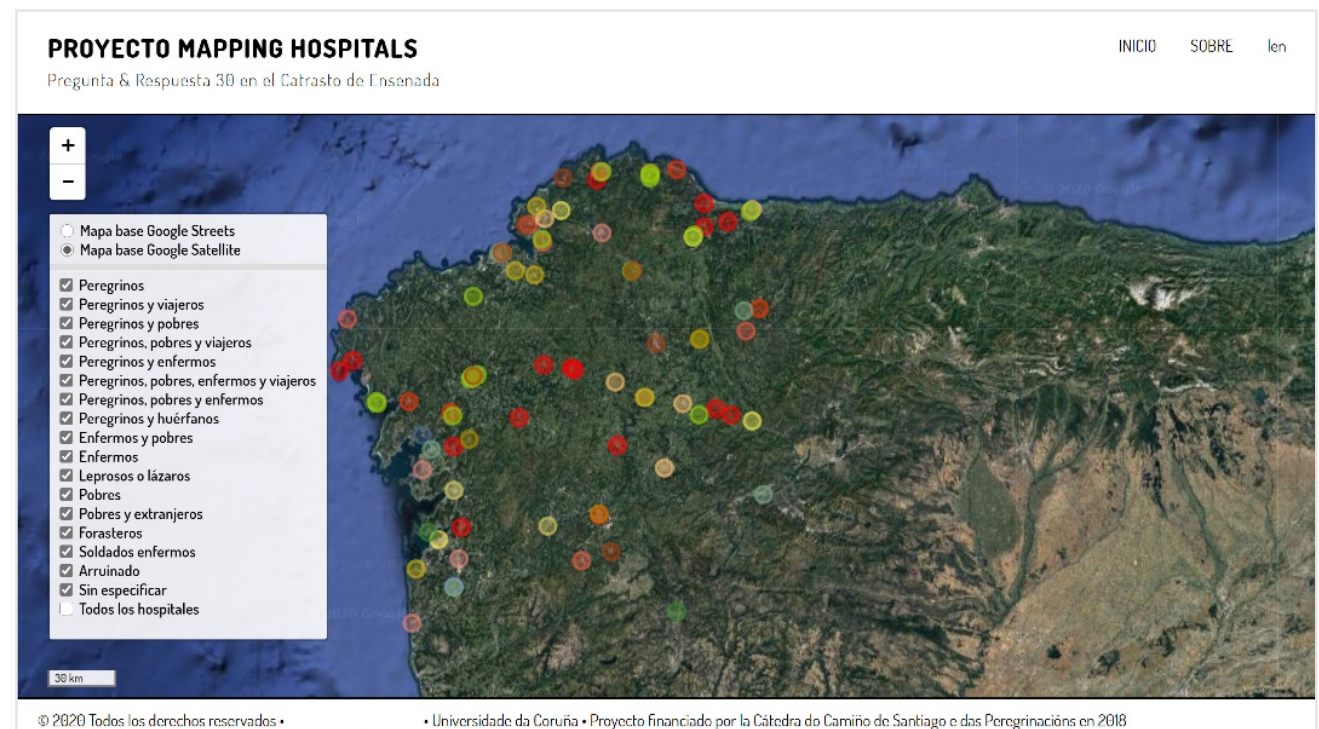

Fuente: Elaboración propia.

Acceder e interactuar con las diferentes capas que configuran este mapa digital es posible a través de un menú despegable situado en la parte superior izquierda del mismo. Al hacer clic en este, el usuario verá listados dos grandes grupos de capas, de las cuales no todas se muestran por defecto al abrir el sitio web (Figura 6). Las situadas en la parte superior del menú son los mapas base. Para este fin se utilizó Google Maps, las vistas Mapas y Satélite, es decir, dos cartografías actuales sobre las que los hospitales están georreferenciados, aunque podrían implementarse otras en el futuro, contemporáneas o incluso históricas. En cualquier caso, de este primer grupo de capas, actualmente sólo una puede ser visualizada, a elección del usuario, pero es fundamental para leer cada arquitectura o un conjunto de ellas en relación con el

13 Inicio del sitio web del proyecto Mapping Hospitals en su versión en castellano, $<$ http://mappinghospitals.udc.es/index_es.html>, con el menú de capas desplegado. 
territorio, con la parroquia o con la trama de la ciudad, villa o pueblo en la que se ubicaban.

Figura 7. Arriba, ejemplo de interacción con un hospital concreto a través de las ventanas emergentes en el sitio web del proyecto Mapping Hospitals. Abajo, modelo de ficha digital individual en el sitio web del proyecto Mapping Hospitals, que da acceso a los atributos asociados a cada hospital geolocalizado.

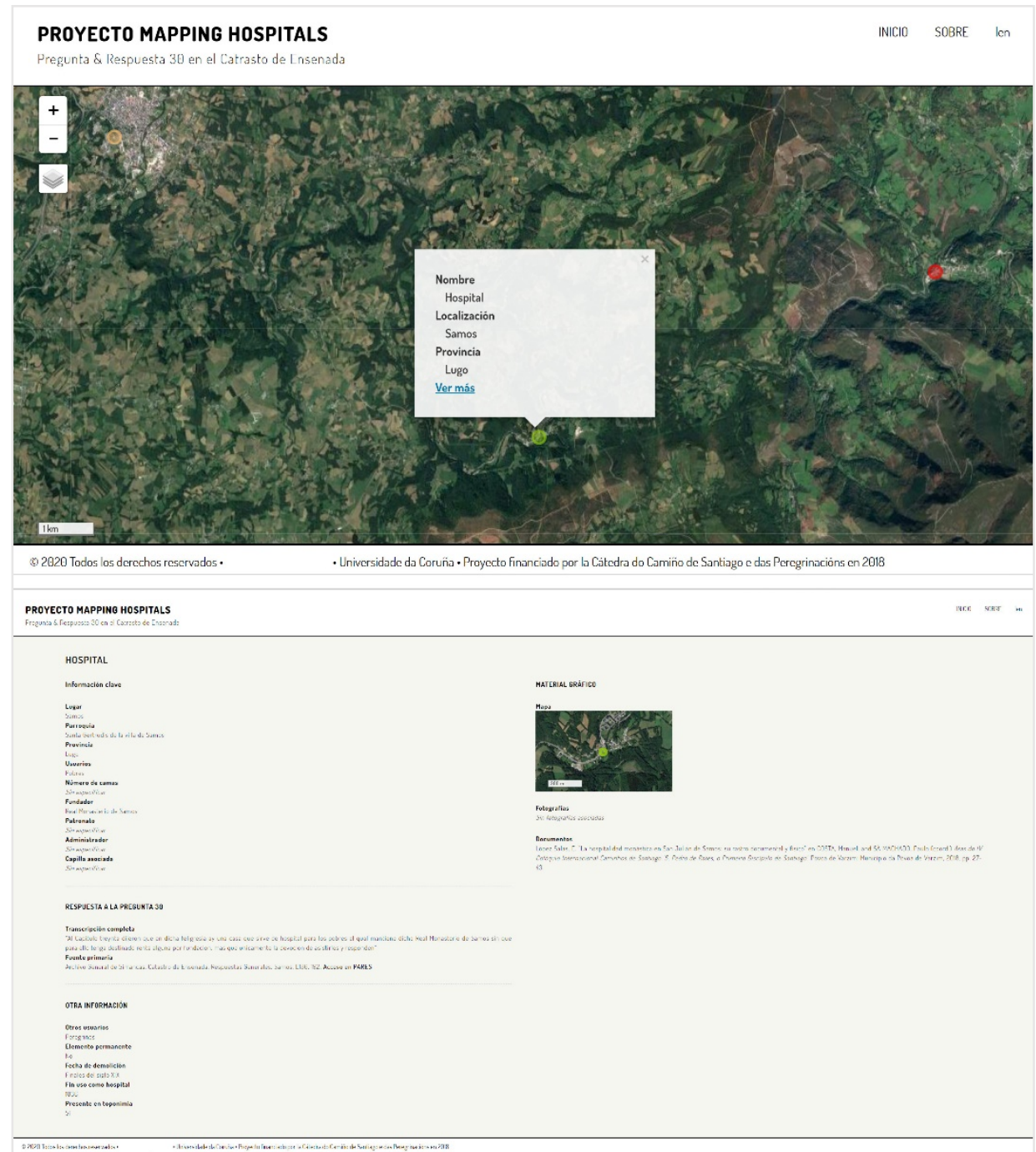

Fuente: Elaboración propia. 
Dentro de este mismo menú, separadas por una línea gris, se despliega un segundo grupo de capas debajo del anterior. Cada una de estas contiene los hospitales clasificados de acuerdo al tipo o tipos de usuarios que atendían, más una última capa en la que es posible visualizar todos los hospitales a la vez, independientemente de a quién estaban destinados. En este segundo grupo, el usuario tiene la posibilidad de hacer que se muestren en el mapa una o varias capas al mismo tiempo, con lo que se abre la puerta a análisis comparativos para un aspecto concreto de los datos recolectados.

No obstante, el mapa digital no sólo permite interactuar con el conjunto, sino también con cada una de las arquitecturas de forma individual. Al hacer clic en un determinado punto se despliega una ventana emergente con tres descriptores sobre el hospital seleccionado: nombre, localización y provincia. En último lugar, se incluye un link titulado Ver más que, si se clica, nos da acceso, en una nueva página web, a todos los atributos que están asociados al hospital en el corpus textual (Figura 7). Bajo el nombre del hospital que figura en el catastro de Ensenada, se relaciona la información clave extraída sobre este: lugar, parroquia, provincia, usuarios, número de camas, fundador, patronato, administrador y capilla asociada. A continuación, el usuario puede leer la transcripción completa de la respuesta a la pregunta número 30 , de la que se extrajeron todos los datos desplegados en la sección de información clave y la referencia a esta fuente primaria dentro del Portal de Archivos Españoles. El último apartado de la zona izquierda de esta ficha digital individual de cada hospital está destinado a acoger otra información recolectada sobre algunos hospitales a través de fuentes o trabajos diversos, como: otros usuarios, si es un elemento permanente o no, su fecha de demolición, el fin de su uso como hospital, si hay pervivencia en la toponimia de su anterior existencia,... Hasta la fecha, este apartado sólo está cubierto para un hospital del corpus, pero irá creciendo en futuros desarrollos del proyecto.

Además de datos textuales, en la zona derecha de estas fichas individuales se consideró importante la presencia de elementos gráficos que, en primer lugar, mantengan a la vista del espectador la localización de la arquitectura en su entorno, a través de un mapa, a una escala de mayor proximidad que en el visor general del sitio web. Inmediatamente debajo, se ha previsto un espacio para incluir fotografías, actuales o históricas, de la fábrica hospitalaria en cuestión. Finalmente, aparece un apartado en el que se indican los documentos que fueron utilizados para ampliar la información ofrecida por el catastro que, entre otras cuestiones, nos pueden ayudar a corregir los errores, ambigüedades, incertezas o ausencia de datos en él detectados durante la investigación.

\section{Conclusiones y líneas de trabajo futuras}

Desde una escala geográfica, en este proyecto intentamos analizar la hospitalidad a través de los edificios dispersos en el territorio, con un trabajo que combina métodos digitales de mapeado web interactivo con fuentes textuales, para obtener una comprensión integral del complejo sistema hospitalario de la región. Nuestra principal 
y más importante contribución es, hasta la fecha, la creación de un mapa web interactivo que permite navegar entre las piezas de patrimonio hospitalario al servicio de la caridad en una escala de aproximación de conjunto, que no elude la individual. De esta forma, las arquitecturas de la hospitalidad recopiladas en el corpus textual del setecientos se hacen interactivas, multi-escalables, interconectadas y enriquecidas en un contexto on-line.

No obstante, el proyecto no sólo se enfrenta a cuestiones de escala, sino también de datos. De hecho, aunque inicialmente concentra su foco de atención en un ámbito muy concreto, el del Reino de Galicia, en el futuro se pretende extender a todo el territorio catastrado a mediados del XVIII, es decir, el de la Corona de Castilla. Asimismo, no se descarta la incorporación de nuevas fuentes documentales que permitan un crecimiento del corpus textual base y de los recursos y posibilidades de análisis que ofrece la primera versión del mapa digital interactivo del proyecto, también con la implementación de nuevos controladores en el visor web.

Este mapa muestra la red hospitalaria en un momento del tiempo concreto, pero con el uso de otras fuentes también se podrían visualizar las redes existentes antes y después de mediados del siglo XVIII, conectadas por los caminos de peregrinación, que estas arquitecturas, en parte, vemos que dibujan sobre el territorio. En otras palabras, el añadir una escala de tiempo al proyecto implicaría un aumento de las posibilidades de descubrimiento en torno a este patrimonio.

En este trabajo, hemos explicado el proceso desarrollado para llegar al mapa interactivo final que, de forma simplificada, se reduce a los siguientes pasos: lectura de las respuestas generales, creación de la base de datos, georreferenciación de los hospitales e implementación del mapa web. Con este último está elaborada una primera base para el análisis de la red, entendida como conjunto de elementos organizados para un fin concreto. Los caminos son elementos clave de la persistencia de la red en el tiempo, porque ponen a los hospitales en conexión. No obstante, el fin común de estas arquitecturas, el caritativo, es también otro elemento que determina la red, así como que la distancia entre ellas no supere, entre un hospital y otro, aquella que se puede recorrer a pie, para volver a encontrar a la noche un cobijo, cuestión de extrema importancia para el peregrino que el relato de Nicola Albani, con el que comenzamos este trabajo, expresa tan bien. Algunos resultados de la lectura de conjunto, del cambio de escala en el estudio de los hospitales son inmediatos y los hemos revelado, pero el significado histórico y arquitectónico de esta aproximación creemos que será una derivada de la continuación del proyecto.

\section{Referencias bibliograficas}

Albani, N. (2007): Viaxe de Nápoles a Santiago de Galicia. Santiago de Compostela, S.A. de Xestión do Plan Xacobeo.

Alimento, A. (2002): Los catastros del siglo XVIII, entre tradición y modernidad. CT: Catastro, 46, 17-26. 
Arribas, M. C. (2001): Caminaron a Santiago... y lo contaron. Crónica en tiempo presente de peregrinos del pasado. Bibliografía Jacobea, Centro de Estudios y Documentación del Camino de Santiago, 2, 4-6.

Camarero, C. (2002): El Catastro de Ensenada, 1745-1756 diez años de intenso trabajo y 80.000 volúmenes manuscritos. CT: Catastro, 46, 61-68.

Castro, R. (2019): Cartografía digital de Galicia en 1753. Jurisdicciones, provincias y Reino. Santiago de Compostela, Andavira.

García, J. J. (1996): Beneficencia y sanidad en el siglo XVIII el hospital de San Juan de Dios de Murcia. Murcia, Universidad de Murcia.

Gijón, V. (2017-2018): Los viajeros extranjeros y los hospitales españoles de la última década del siglo XV hasta la revolución francesa. Vectores de Investigación, 12-13, 259-308.

González, M. I. (1993): Viaje de Nápoles a Santiago de Galicia. Madrid, Edilán para Consorcio de Santiago.

González, M. I. (2018): Cosimo III de’Medici y N. Albani (distintas sensibilidades). Revista de la Archicofradía Universal del Apóstol Santiago, 60, 40-46.

Herbers, K. y Plötz, R. (1998): Caminaron a Santiago. Relatos de peregrinaciones al "fin del mundo". Santiago de Compostela, Xunta de Galicia.

Lois, R. C. y López, L. (2015): El origen del turismo viajero italiano a lo largo del Camino de Santiago. Investigaciones Turísticas, 9, 132-159.

Rodríguez, M. (2004): La información en el sistema de gobierno y administración de la Monarquía en la primera mitad del siglo XVIII. En Serrano, E. (Ed.): Felipe V y su tiempo: congreso internacional, vol. 1. Zaragoza, Institución «Fernando el Católico» y CSIC, 947966. 